\title{
OBSERVATIONS OF STELLAR SPECTRA \\ RELATED TO EXTENDED ATMOSPHERES
}

\author{
K. O. WR IGHT \\ Dominion Astrophysical Observatory, Victoria, B.C.
}

\begin{abstract}
Although the Struve Symposium was planned to discuss conditions in binary stellar systems, it is necessary to consider the observations of extended atmospheres in single stars in order to compare them with those of binary systems. The Sun, being the closest star, has the first chromosphere that was observed, and we can assume that the many features observed on the Sun will be much enhanced in giant stars with more extended atmospheres. Among the early-type stars, the hydrogen emission lines in the spectra of Be stars are known to vary and this suggests the atmospheres are unstable. Similar, even more pronounced effects, are seen in the spectra of shell stars such as 48 Librae. The supergiant stars have also been shown to have spectral lines that change, often quite rapidly, with time. Smolinski has observed in the spectra of some F and G-type supergiants much-broadened absorption lines that also vary with time. Wilson's systematic studies of the $\mathrm{H}$ and $\mathrm{K}$ emission lines in late-type stars have greatly extended our knowledge of stellar chromospheres, and the ionized magnesium lines at $2800 \AA$ should give additional information as more extra-terrestrial observations are obtained. Numerous observations of the $\zeta$ Aur stars have been obtained near eclipse; $\zeta$ Aur and 31 Cyg seem to be similar systems and show similar phenomena - a rise in temperature outward from the photosphere and an extensive, variable calcium chromosphere, but a viable theory for these chromospheres has not yet been given. VV Cep and $32 \mathrm{Cyg}$ probably are larger and more complex systems. The emission lines in the ultraviolet spectra of the M-type stars may be indicators of coronae in these cooler atmospheres. Most variable stars seem to have extended atmospheres and, when detailed studies are made, show changes in both light and spectrum. Shock-wave phenomena are undoubtedly important in the analysis of many of these stars, as also are fluorescent, selectiveexcitation processes. Flare stars, though generally dwarfs, are of great interest in studies of stellar atmospheres.

Extended atmospheres can be detected from observed gross deviations from local thermodynamic equilibrium, as in the study of emission lines, large velocity fields, and dilution effects. It is probable that the presence of a companion affects the atmosphere of the primary star in close double stars, but in stars like $\zeta$ Aur and $31 \mathrm{Cyg}$, the atmospheric effects produced near eclipse may be among the best clues for the interpretation of extended stellar atmospheres.
\end{abstract}

The study of binary stars has progressed rapidly in recent years, from the observations of light and radial-velocity variations and their analysis in terms of simple binary motion to the detailed analysis of the light curves in terms of the physics of stellar atmospheres and the radiation and gravitational effects of one star on the other, and to the detailed study of spectrum variations that are the result of these interactions. The spark for much of the latter type of investigation came from the work of Otto Struve who utilized the time he had on the McDonald telescope thirty years ago to observe with great efficiency the most interesting eclipsing binary stars, and to lay the foundations for our symposium here at Parksville.

We have heard a great deal about Struve's work, and about more recent work with other spectrographs which only now can improve on Struve's observations. However, it is desirable that we review again the observational data related to extended stellar 
atmospheres in order that we may distinguish, if possible, the data obtained from observations of double stars, in which the components are interacting, from phenomena observable in normal stellar atmospheres. The analysis of the basic spectroscopic observations is difficult and the decision whether the observed lines come from the photosphere, an extended atmosphere, an expanding atmosphere or from matter moving between the stars is often most challenging. Just as Plavec (1967) has asserted that nearly all spectroscopic binaries must be considered 'close' by his definition, so Thomas (1970) and his colleagues have come to the conclusion that a stellar atmosphere has an almost limitless extension and that for our dwarf Sun, even the solar wind, which affects the planets, is part of this atmosphere.

Therefore, in this review, I feel that we can consider any observational topics that have not yet been discussed. I should like to consider stellar chromospheres as the principal subject of this review, but will mention other types of outer atmospheric phenomena in case some of the participants wish to talk about them later. These will cover the extended atmospheres of early-type stars, supergiants of all types, and something about the many kinds of variable stars, including the flare stars which seem to be dwarfs. Mass loss in general has already been discussed in previous sessions, as have expanding envelopes of all types, but especially the large-scale expansions observed in some of the hot B-type stars observed by Morton and others in the far ultraviolet region of the spectrum. It is hoped that by recording data for normal stars it will be possible to separate such data from those that are peculiar to and the result of binary interactions. I should mention a few earlier conferences related to this subject: The Sun Among the Stars, Riverside, Cal. 1966 (unpublished); Spectrum Formation in Stars with Steady State Extended Atmospheres, Munich, 1969, NBS Special Publ. 332 (H. R. Groth and P. Wellmann, eds.) and Stellar Chromospheres, Goddard, IAU Colloq., No. 19, February 1972 (to be published). There have been many reviews related to extended stellar atmospheres, but I shall mention only a few in addition to the above - Feast (1970), Deutsch (1970) and Thomas and Gebbie (1971).

The chromosphere of the Sun, of course, was the first observed; it was seen as an emission-line spectrum at the eclipse of 1870 . The importance of observing the solar chromosphere as a means of studying the structure of the outer atmosphere was quickly recognized, and great efforts were made to obtain flash spectra at each favourable eclipse, especially by the British Royal Society expeditions, and those by Mitchell in the United States. Pannekoek and Minnaert (1927) analyzed the 1924 eclipse data and Menzel (1931) the Lick eclipse data, but the analysis of the Khartoum 1952 spectra by Thomas and Athay (1960) and their colleagues has given us the greatest insight into conditions in the 'medium' layers of the solar atmosphere. The helium lines, especially that at $\lambda 10830$, the emission of the ionized calcium lines, $H$ and $K$, and, of course, the corona are now well-documented evidence for the extended atmosphere of the Sun; the studies of Linsky $(1968,1972)$ are among the most important papers outlining investigations of the solar calcium lines. Yet, according to all other evidence, including its mass and luminosity, the Sun is a dwarf star and one would 
therefore expect that its outer atmosphere would be less extensive than those of the vast majority of stars of greater luminosity. Lower level effects, such as sunspots, granulations, plages, etc., are phenomena that may be common in stars, though they would be difficult to observe, and they may produce some of the line-broadening effects, but they are not applicable to our present discussion. However, prominences and spicules do extend above the 'normal' photosphere and as such could be introduced as topics for discussion at the appropriate time. We shall not discuss the Sun further here, but will consider it only as a basis for comparison with other stellar atmospheres. In this connection, I agree with Thomas who has always tried to develop theories applicable to the Sun and extend them to the stars.

\section{Early-Type Stars}

Underhill (1966) has covered the observed data for early-type stars in her book on the subject, and she has also noted the salient features of these stars that can be related to extended atmospheres in her survey paper at the Munich Colloquium (1970). Under this topic we shall consider all stars earlier than type F, since this class is where the convective zone seems to appear and where large rotational velocities almost disappear. We shall not go into detail concerning the relevant observations for each type of star, but will mention in this category Wolf-Rayet stars, Of stars, $P$ Cygni and other $B$ and $O$ supergiants, Be and shell stars.

Most of the $\mathrm{Be}$ stars have emission lines in their spectra that are produced in a rapidly rotating envelope surrounding the main star, and these lines also have a central absorption core. The hydrogen lines are usually the strongest lines in the spectra of Be stars, with $\mathrm{H} \alpha$ having much the strongest emission and other lines in the Balmer series becoming successively weaker with the result that by $\mathrm{H} \gamma$ and $\mathrm{H} \delta$ the emission is often only a double hump near the centre of the strong absorption line. The Burbidges (1953) showed that the hydrogen emission is a recombination spectrum modified by self absorption and by electron scattering. These atmospheres are unstable since the emission in many stars changes with time, the most frequent change being a variation of the relative strengths $(V / R)$ of the two emission lobes, with a resultant change in the observed radial velocity of the central absorption core. Struve and others concluded that the angular momentum of these stars is conserved and, from measurements of the rotational velocities, found that the extended atmospheres had radii about $3 R_{\odot}$.

Probably most of the hot O-type stars have extended atmospheres. The Of stars usually show emission at $\mathrm{H} \alpha$ and at CIII, $\lambda$ 5696; some show He II emission at $\lambda 4686$, as do lines of Niv, Nv, Sirv and HeI. 9 Sagittae, O7f, may be considered a typical star of this class, and observations of its spectrum indicate that the emitting shells are variable and moderately extended. Some of the lines may be excited by the Bowen fluorescence mechanism by lines at and below the Lyman continuum.

The very hot Wolf-Rayet stars, interesting though they are, will not be discussed in detail here since they have been the subject of two recent conferences, at Boulder 
in 1970 and at Buenos Aires in 1971. The discussion about the binary character of Wolf-Rayet stars has not yet been completely resolved, though a number of these stars are certainly components of binary systems. It has been suggested that there are two types of these objects, though they in turn are subdivided into the carbon and the nitrogen sequences. The masses seem to be between $5 M_{\odot}$ and $10 M_{\odot}$. Underhill has proposed, as a model for a single Wolf-Rayet star, a photosphere with a continuous spectrum like that of an $\mathrm{O}$ star with radius $7 R_{\odot}-10 R_{\odot}$, an inner compact atmosphere where the rounded-top emission lines are formed, of thickness $1 R_{\odot}-2 R_{\odot}$, and an outer, expanding, low-density atmosphere, where dilution effects and monochromatic fluorescent effects occur with a radius, if V 444 Cygni is typical, of $17 R_{\odot}$.

Typical shell stars seem to have B-type central stars. They were studied extensively by Struve and he was probably the first to discuss dilution effects, which make lines arising from metastable levels stronger in these low-density envelopes than the other lines produced in the photosphere. Struve and Wurm (1938) commented on the great strength of the metastable lines of FeII, NiII, CrII, etc., relative to $\mathrm{Mg}$ II, $\lambda 4481$. One feature of shells is that the spectra correspond to conditions of lower temperature and lower density than the underlying absorption lines, as might be expected. Thus shell lines are usually deep and moderately sharp, somewhat similar to those in $\alpha$ Cygni (A2p), though the underlying spectrum may be that of a B star. In the early studies of shell stars it was not realized that the shells are unstable and variable. However, Merrill and Sanford (1944) noted variations in the spectrum of 48 Librae beginning in 1938. Its spectrum has since varied in a period of about $10 \mathrm{yrs}$ (Underhill, 1966). The radial velocities all follow the same general trend but the hydrogen, Fe II and TiII lines have successively greater ranges corresponding to high, intermediate and low levels in the atmosphere. In stars like these, the line profiles are often asymmetrical, with the asymmetries on the side of the line to which the velocities are progressing. There is also a progression of velocities for the hydrogen lines, with the highermember weak lines showing larger variations, which are interpreted as formation in the deeper layers of the atmosphere. Another shell star with a long history is $\gamma$ Cassiopeiae. Hutchings (1970), during the course of his investigations of stars with rotationally extended envelopes, has found short-period changes in the emission line profiles with a period of 0.7 , but over the years its brightness has varied by more than a magnitude, and its shell has appeared and disappeared.

Supergiant stars are usually considered to have extended atmospheres. The data were surveyed and up-dated at the 1971 Trieste Colloquium on Supergiant Stars. The early-type supergiants are considered here separate from those of later types because of the Wilson-Bappu effect, among other considerations. Underhill has noted that supergiants earlier than $\mathrm{O} 9$ cannot exist because their effective gravity would be too low $\left(\sim 5 \times 10^{3}\right)$ to balance the large radiative pressure gradient. P Cygni has usually been considered a prototype for early-type supergiants with its fairly sharp emission lines and strong, shortward-displaced absorption lines; it may not be typical since its luminosity has changed by several magnitudes over the centuries, but it does seem to be a fact that the supergiants do vary in light and also show small variations in radial 
velocity. In P Cygni itself, N II and He I lines have moderately strong emission, while N II and Si IV lines, which have higher ionization potentials, appear only in absorption. Ghobros (1962) agrees with the estimate by Struve and Roach (1939) from dilution effects that the radius of the expanding shell is about 2.5 times the stellar radius. For other B-type supergiants, Chentsov and Snezhko (1972) have studied $\beta$ Orionis (B8Ia) and have found differential velocity shifts of about $10 \mathrm{~km} \mathrm{~s}^{-1}$ for $\mathrm{Hel}$ and ion lines, and a Balmer progression of $10 \mathrm{~km} \mathrm{~s}^{-1}-20 \mathrm{~km} \mathrm{~s}^{-1}$ similar to that observed for shell stars. Hutchings (1971) has recently obtained high-resolution line-profile scanner observations of B- and A-type supergiants and found that these stars have outwardaccelerating envelopes and that the profiles change with time, sometimes in a few hours. There is a velocity-excitation relation with a velocity reversal which indicates a temporary, unstable atmospheric structure. The general picture agrees with other observations that there may be pulsations that propogate and weaken outwards from the stellar surface. He suggests that the A-type stars have evolved from mass-loss B-type stars. Rosendahl (1970) studied line profiles in 64 B- and A-type Ia and Iab supergiants. He concluded that both rotation and macroturbulence contribute to the line broadening, with rotation the principal factor up to middle B types, and turbulence becoming more important thereafter. He concluded that, though the stars may be losing small amounts of mass, there is little evidence for loss of angular momentum. Since the spectrum of $\alpha$ Cygni is often referred to in shell spectra, reference should be made to the analysis by Groth (1960), who obtained $T_{\text {eff }}=9170 \mathrm{~K}$, and gravity, $\log g=1.13$, assuming radiative equilibrium except for the outermost layers of the atmosphere; the line widths and intensities correspond to a macroturbulent velocity of $22 \mathrm{~km} \mathrm{~s}^{-1}$ and a microturbulent velocity of $7.8 \mathrm{~km} \mathrm{~s}^{-1}$ in the lower layers and $20 \mathrm{~km} \mathrm{~s}^{-1}$ in the outer regions; the radial velocity variations suggest small irregular pulsations, and the atmosphere in general seems to be similar to the lower solar chromosphere.

In his article on supergiants and Cepheids, Kraft (1960) considered that line profiles in spectra of A- and early F-type stars of luminosity classes Ib and II could be explained by a combination of microturbulence and rotation (which was confirmed later by Rosendahl, 1970) assuming the conservation of angular momentum as stars evolved across the top of the $\mathrm{H}-\mathrm{R}$ diagram. He also found that Cepheids with periods of less than $10 \mathrm{~d}$ also fitted this hypothesis; for G- and K-type stars of class Iab, and Cepheids with periods more than $10 \mathrm{~d}$, large-scale turbulence may be more important than rotation. There is an additional absorption at $\mathrm{H} \alpha$ and a few other lines in the spectra of some Cepheids; the $\mathrm{H} \alpha$ satellite is similar to that observed in 89 Herculis (F8 Ia, Böhm Vitense, 1956), where hydrogen and sodium components appear, though the displacements are different. The study of Cepheid variables as examples of extended atmospheres is certainly valid, but because of their position in an instability area of the H-R diagram which, presumably, is the reason for the pulsations, they cannot be considered normal stars. Other F-type supergiants that show evidence for mass motions by the appearance occasionally of double absorption line components are $\varrho$ Cassiopeiae, F8 Iap (Bidelman and McKellar, 1957) and RW Cephei, G0 Ia 
(Merrill and Wilson, 1956). More recent work on F0 to K5 supergiants by Smolinski (1972) has shown that a few Ia stars have H $\alpha$ emission; in HD 217476, [N II] emission has also been observed. Other stars, including HD 231195, F5 Ia, and HD 12399, G5 Ia have lines that seem to be turbulence broadened and that are twice as broad as those in $\delta$ Canis Majoris; some lines also show evidence of variable structure.

\section{Late-Type Stars}

For the late-type stars, Deutsch (1970) at the Lunteren Symposium summarized most of the data concerning chromospheric phenomena. He reviewed the observations that the hydrogen lines vary in many giants and supergiants, and that there does not seem to be a direct correlation with changes in the $\mathrm{CaII} \mathrm{H}$ and $\mathrm{K}$ lines. Adams and Russell (1928) had noted the strengthening of the Balmer lines relative to the metals, which was confirmed for $\alpha$ Orionis, M2 Iab, by Spitzer (1939) when he found $T_{\text {exc }}=2100 \mathrm{~K}$ for iron lines and $17000 \mathrm{~K}$ for the Balmer lines; the hydrogen lines are probably formed in a high-temperature region similar to the solar chromosphere. Time variations in the $\mathrm{Ca} I \mathrm{H}$ and $\mathrm{K}$ lines have been observed in several $\mathrm{K}$-type stars by Griffin (1963), Liller (1968) and others. A period of $350 \mathrm{~d}$ for such variations in $\alpha$ Tauri, suggested by Kraft (1967) would fit the hypothesis that $\alpha$ Tauri has evolved from a main-sequence A-type star which has lost its angular momentum through a stellar wind. Further evidence for chromospheric activity in late-type stars is the presence of helium lines in some spectra. Wilson and Aly (1956) identified the He I absorption line at $\lambda 5876$ in the spectra of several stars that had strong $\mathrm{H}$ and $\mathrm{K}$ emission. Vaughan and Zirin (1968) have observed the helium line at $\lambda 10830$ in the spectra of a 'substantial' number of the 75 F- to M-type stars they observed. The absorption intensity increases in general with the intensity of the $\mathrm{Ca}$ II $\mathrm{K}$-line emission; it appears in emission in a few stars. It is not present in F- or M-type stars. The observations suggest that the helium line indicates the presence of a hot chromosphere, and that it originates in discrete clouds or streams rather than in a homogeneous layer.

Much of our knowledge about stellar chromospheres has come from Wilson's work on the $\mathrm{CaII} \mathrm{H}$ and $\mathrm{K}$ lines. The basic results were given by Wilson and Bappu (1957), who found that these lines appear in emission in nearly all giants and supergiants of spectral types $\mathrm{G}, \mathrm{K}$ and $\mathrm{M}$ and that there is an almost linear relation between $\mathrm{K}$-line emission width and luminosity over a range in absolute magnitude from $M_{v}=+10$ to $M_{v}=-6$. Similar emissions are observed in the spectra of some main sequence stars of types as early as F5, and the frequency increases to later types. The lines are usually double-peaked, as in the spectrum of the Sun, though they cannot be seen in the solar spectrum at the dispersions used for the stars; thus it is presumed that chromospheres similar to the Sun are present also in the stars. As Deutsch (1970) notes, the intensities of the peaks vary, and fine structure in the $\mathrm{K}$ lines is observed in the spectra of many red giants, from which prominence activity can be inferred. An excellent summary of our knowledge of stellar chromospheres, based on these data, is given by Wilson (1966). With the advent of observations beyond the Earth's atmo- 
sphere, much more information about stellar chromospheres can be obtained, though the resolution for most of the observations to date is quite low. Doherty (1971) has shown that the MgII doublet, $\lambda \lambda 2795,2802$ can be seen at a resolution of $25 \AA \mathrm{mm}^{-1}$ for giant and supergiant stars later than K2, and Kondo et al. (1972) have obtained rocket observations of these lines for five stars with a resolution of $7 \AA \mathrm{mm}^{-1}$. The latter find a fairly good correlation between the $\mathrm{Mg}$ II line widths and the CaII data of Wilson and Bappu; the widths of the $\mathrm{Mg}_{\mathrm{II}}$ lines are definitely broader than those of Ca II, possibly as a result of the greater abundance of magnesium.

Wilson concludes from solar data that the chromosphere is not in hydrostatic equilibrium, that it and the corona are made up of material expelled upward from below, and that they are dynamic rather than static structures. The most likely source of the required energy is the convection zone where the hydrogen is partially ionized and where the energy flux is carried by the turbulent mass motions of convection. The turbulent elements generate acoustic waves which, as the energy decreases, become magnetohydrodynamic, and are thus related to magnetic fields which produce shocks to dissipate energy into the chromosphere. Wilson surmizes that all stars with surface temperatures lower than the Sun have convective zones. The observations that chromospheric $\mathrm{K}$ lines are observable only as early as the late F-type stars, and that stellar rotation decreases markedly at the same types, are used as the basis for the conclusion that nearly all late-type stars have chromospheres. For a number of years, Wilson has been expanding his interpretation of these results and the relation between luminosity and the width of the $\mathrm{K}$ line, to determine its connection with the age of the stars, the masses of evolved stars, and the rate of star formation.

\section{The $\zeta$ Aurigae Stars}

Probably the most detailed information concerning the outer atmospheres of late-type giant stars should be obtained from eclipses of these stars when they are observed just prior to, and just after, total eclipse. The best examples are the $\zeta$ Aurigae stars. The principal component of these systems is a supergiant $\mathrm{K}$ - or M-type star and the secondary component is a main-sequence, early-type $\mathrm{B}$ or $\mathrm{O}$ star. The best data available are for $\zeta$ Aurigae (Wilson and Abt, 1954; Groth, 1957), for 31 Cygni (Larsson-Leander, 1957; Wright, 1959) and for VV Cephei (Goedicke, 1938; Peery, 1966). Wilson (1960) has summarized the available publications to 1958; Wright (1970) has discussed the chromospheric $\mathrm{K}$ line with its observed multiple structure for these atmospheres, and Groth (1970) has considered the data from the point of view of steady-state extended atmospheres.

The basic observations for these stars are fairly well-known, though more data are needed, and, probably, new analyses should be made in order to clear up the present uncertainties in the interpretation of the results. There is an inner chromosphere, that can be defined as the region where chromospheric absorption lines of neutral and ionized metals can be observed near eclipse. These lines are superposed on the composite spectrum of the two components and are produced by absorption in the inner 
chromosphere as the radiation from the early-type star passes through the extended late-type atmosphere. These lines can be observed from about $3200 \AA$ to $4500 \AA$, where the energy from the late-type star becomes markedly greater than that from the early-type star; the lines can be detected to about a stellar radius from the limb (defined as second and third contacts of the eclipse). The strong ionized metallic lines can be observed somewhat farther from the limb, and multiple structure in the TiII lines at $3759 \AA-61 \AA$ can be seen at times. The CaII $\mathrm{H}$ and $\mathrm{K}$ lines have been detected nearly five stellar radii from the limb in 31 Cygni (Wright and Odgers, 1962). Multiple components of these lines have been detected on high-dispersion spectra that have been obtained at each eclipse since they were first observed for 31 Cygni at Victoria (McKellar et al., 1952). These components are sometimes well separated and almost equal in intensity to the principal component, but more often are weak and barely distinguishable from the grain of the plate. The intensities of the Ca II lines do not necessarily follow the same pattern from eclipse to eclipse (Wright, 1970), which suggests that the outer atmospheres may vary in extent as well as in density. Certainly the multiple components suggest large-scale activity, such as prominences or condensations of some kind.

The increase in temperature outwards in the inner chromosphere has been found from curve-of-growth studies of the chromospheric lines in both $\zeta$ Aurigae and 31 Cygni and therefore seems to be well documented as similar to the solar chromosphere. The intensity data on which these temperatures are based are difficult to derive accurately because of the complexity of the K-type spectrum on which the chromospheric lines are superposed, and because the relative intensities of the continua of both stars are required (although the continuum of a K-type star is difficult to define) for the determination of the chromospheric intensities. The interpretation of the atmospheric densities is still uncertain since it is not known whether the source of the excitation in the chromosphere is from the B star or from some 'super-excitation' phenomenon in the outer atmosphere of the $\mathrm{K}$ star. Both interpretations seemed to required large clouds rather than a smooth density distribution in the outer atmosphere to explain the observed ionization phenomena. Kawabata (1957) studied the same problem, using Wilson and Abt's data, and found that an electron temperature of $12000 \mathrm{~K}-14000 \mathrm{~K}$. might satisfy the data assuming that ionization by electron collisions is more important than radiative processes. More recently, Magnan (1965) has considered departures from LTE for a three-level model for the CaII atom and found that, with an electron temperature of $15000 \mathrm{~K}$ and $N_{e}=10^{10}$, an atmosphere with homogeneous density can be derived from the Victoria data for 31 Cygni. He suggests that the 'satellite' lines can be produced by large jets or prominences, possibly similar to those in the Sun, which could be cold elements in the hotter, outer chromosphere. The disappearance of the other metallic lines about $0.5 R_{K}$ would be the result of a rapid increase in electron temperature at this height, and thus the cold elements would not be observed.

Motions in the atmospheres of these stars can be obtained from radial-velocity measurements of the chromospheric lines. Near eclipse the velocities of the metallic 
lines definitely deviate from the velocity curve derived from observations obtained far from eclipse. However the velocities do not follow the pattern of the rotation effect, such as is observed for Algol when one star gradually passes behind the other. While the velocities do not seem to be entirely random, since at a given eclipse, they tend to be chiefly positive or negative before and/or after totality, the pattern for different eclipses and for different stars does not seem to remain the same. As Wilson (1960) notes, the radial velocities probably indicate a combination of random and systematic motions of gases in the chromosphere. Saito (1970) made a most detailed study of the velocities observed for $\zeta$ Aurigae and concluded that the egress velocities could be explained as a combination of ejection from the chromosphere superposed on a slow rotation and non-steady motion of the gas clouds.

The general motions in the atmospheres of these stars can also be inferred from the microturbulent velocities derived from curves of growth and macroturbulent velocities obtained from the line profiles. The microturbulent velocities found by Wilson and Abt (1954) for $\zeta$ Aurigae range from $6 \mathrm{~km} \mathrm{~s}^{-1}$ to $13 \mathrm{~km} \mathrm{~s}^{-1}$; they seem to be slightly greater for ionized than for neutral atoms and the velocities may increase slightly with height in the atmosphere; there is some evidence for similar results from Wright's (1959) analysis of the inner chromosphere of 31 Cygni. Macroturbulent velocities derived from line profiles were calculated by Kitamura (1967) from an ingress plate of $\zeta$ Aurigae and found to be between 15 and $19 \mathrm{~km} \mathrm{~s}^{-1}$. McKellar et al. (1959) studied the K-line profiles of 31 Cygni for the 1951 egress and obtained a good fit with a velocity of $20 \mathrm{~km} \mathrm{~s}^{-1}$ for the lower chromosphere and $10 \mathrm{~km} \mathrm{~s}^{-1}$ for the upper chromosphere.

The dimensions of the $\zeta$ Aurigae stars are summarized by Wright (1970). Although the spectroscopic and photometric data do not entirely agree, the diameters for the late-type components are probably 200 to 1000 solar diameters and their masses are 8 to 20 solar masses; the mass ratios are not greatly different from unity. The separation of the components is from 2 to 10 astronomical units, and thus large-scale effects of the $\mathrm{B}$ star on the $\mathrm{K}$ star should not be expected.

Much work remains to be done on the analysis of these important systems; considerable material has been accumulated at Victoria and elsewhere during the past few years. $\zeta$ Aurigae was favourably situated for observations during its eclipse in mid-winter, 1971-1972 and a good series of observations was obtained by Simon in Hawaii. The system of 31 Cygni just came out of eclipse in July, 1972. The K line does not show as much detailed structure as in 1951 or in 1961, but sharp features superposed on the broader chromospheric feature are easily seen. The spectrum of 32 Cygni shows more supergiant characteristics than those of 31 Cygni or $\zeta$ Aurigae, but the system is not so well suited for precise analysis because the eclipse is almost grazing, and its extent seems to vary from eclipse to eclipse. A good series of plates showing the chromospheric spectra of 32 Cygni was obtained at Victoria in 1968 and are shown in Figure 1. A less extensive series was obtained in 1971; it is hoped to make an analysis of the chromosphere from these data. Similarly VV Cephei is being followed around its orbit beginning with the chromospheric phases in 1956-1959; 


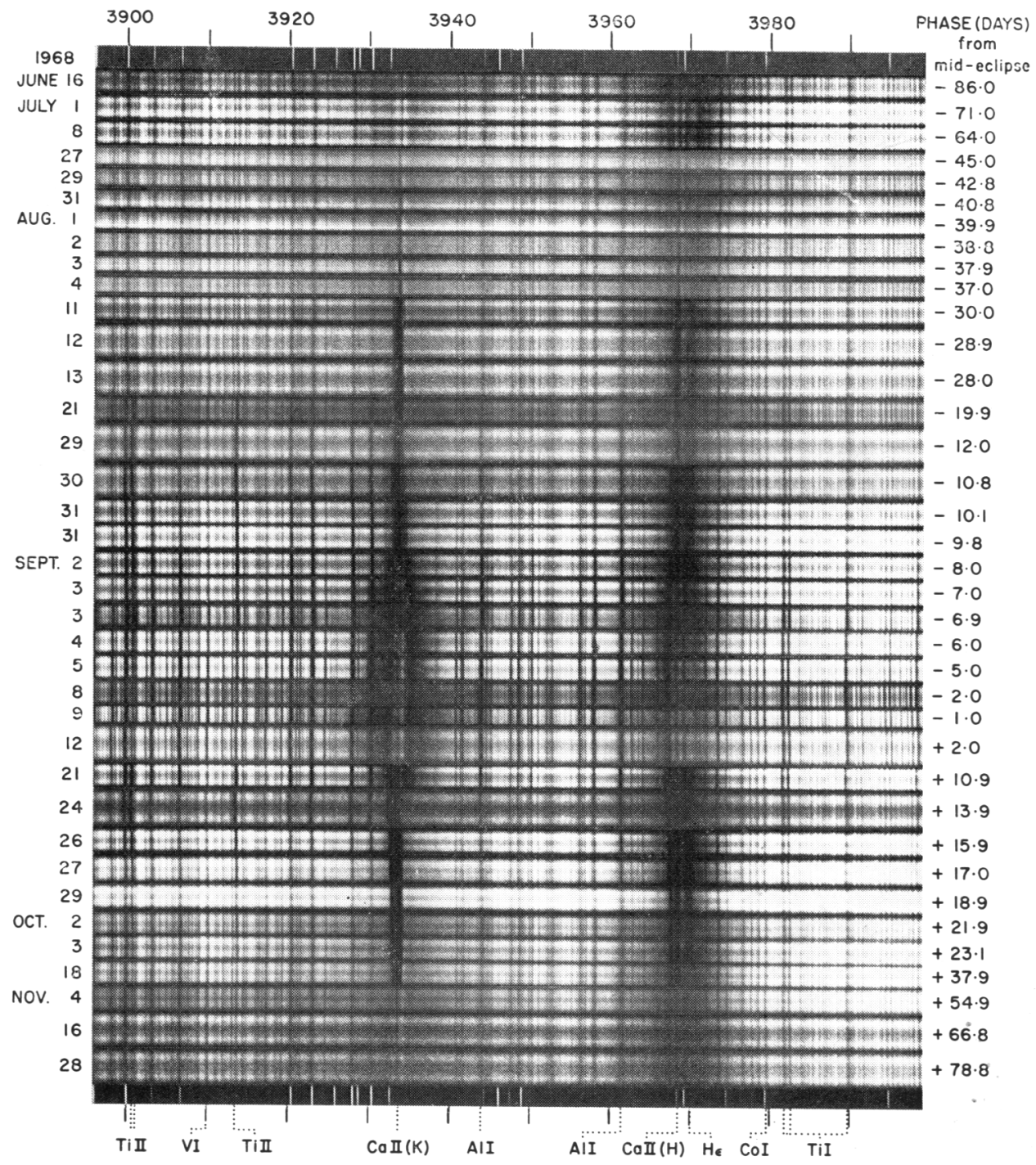

Fig. 1. Victoria Observations of $32 \mathrm{Cyg}$ at the 1968 Eclipse.

These high-dispersion ( 2 to $6 \AA \mathrm{mm}^{-1}$ ) spectra cover the period when changes in the Ca II $\mathrm{K}$ line were observed. Inner chromospheric effects on the lines of $\mathrm{Fe}$ I, Ti II, Al I, H, etc. can be observed from phases about \pm 25 . Mid-eclipse is assumed to be J. D. 2, 440, 109.8 based on photometric observations up to 1971 .

although it does not go into eclipse again until 1976, the chromosphere is so extensive that some effects are expected to appear in a year or two. The velocity curve now seems fairly well defined (Hutchings and Wright, 1971) from which a mass of $20 M_{\odot}$ for each star has been obtained. The system $\varepsilon$ Aurigae has frequently been considered as one of the $\zeta$ Aurigae stars (Wright, 1970). The spectrum certainly shows chromo- 
spheric effects at the time of eclipse, but the dimensions derived from the spectroscopic and photometric data are quite discrepant, and a completely satisfactory model for the system has not yet been found, though many have been suggested.

A number of other systems similar to VV Cephei have been described by Cowley (1969); for a long time, VV Cephei was considered to be 'one of a kind', and its spectrum was considered so complex that it was thought that it would be almost impossible to analyze. The most similar systems seem to be WY Geminorum and Boss 1985, which are being studied by Cowley; it is not yet certain that either system undergoes a total eclipse. The principal feature of these spectra is the 'reverse P Cygni' emission indicating a negative velocity relative to the absorption lines, which has been interpreted by Faraggiana and Hack (1969) in terms of a hydrogen envelope around the hot star which is rapidly rotating and also contracting. However other emission lines, chiefly of ionized metals, from both permitted and forbidden transitions, appear distinctly and with approximately the same velocity, in the ultraviolet region of the spectrum, and are the lines that frequently appear in the spectrum of M-type supergiants. Reverse P Cygni profiles are unusual in stellar spectra, but have been noted by Conti (1972) for the He II $\lambda 4686$ line in the spectrum of $\theta^{1}$ Orionis $C$. They have also been observed by Walker (1972) in YY Orionis stars which are still in the gravitationally contracting phase, and therefore very young.

Many of the symbiotic stars are similar to the VV Cephei stars. For instance, $\mathrm{CH}$ Cygni showed remarkable changes in the past few years, having had outbursts in 1964 and 1967. The forbidden Fe II emission lines seemed to be formed under somewhat similar conditions to those in VV Cephei - but probably in a region far beyond the extended atmosphere that we are discussing (Faraggiana and Hack, 1969) Most observers seem to consider the symbiotic stars as binaries and, in particular, Boyarchuk (1969) has shown that the simplest explanation for these stars is a combination of a giant M-type star with a hot companion, both of which are embedded in a nebula with the hot star providing the excitation for the high-excitation lines. However, according to Boyarchuk's diagram, the Fe II emission lines may be found in the outer atmosphere of the $\mathrm{M}$ star.

The presence of metallic emission lines in the ultraviolet region of the spectra of luminous late-type stars was noted by Herzberg (1948) for $\alpha$ Herculis and $\alpha$ Scorpii. For many years they have been known to appear in the spectra of long-period variables (Merrill, 1960), chiefly as singly-ionized lines, both permitted and forbidden. These are the lines that appear in the symbiotic stars (Sahade, 1960) though in the spectra of some stars such as CI Cygni and AG Pegasi, lines with excitation as high as those in the solar corona have been observed. As Herzberg (1948) observed, in late-type stars, the corona would be expected to have a much lower temperature than that of the sun so that FeII lines rather than those of Fex might become prominent. As noted above, many of the investigations of the ultraviolet absorption and emission lines have been undertaken for the VV Cephei stars (Cowley, 1969). Struve (1944) listed many of the lines in the spectrum of VV Cephei, and Swings (1969) has tabulated the lines identified in the spectrum of Boss 1985 in the region $3144 \AA-3626 \AA$. 
A more detailed study of VV Cephei covering most of the cycle is being carried out by Wright (unpublished). The emission lines may, in some cases such as $\alpha$ Herculis (Deutsch, 1956), be produced around and between the stars, but the spectra of $\alpha$ Scorpii $\mathrm{A}$ and $\alpha$ Orionis seem to indicate that they arise in the outer atmosphere of the $M$ star. Observations of these lines in the spectra of other M-type stars have been reported by Bidelman and Pyper (1963).

\section{Variable Stars}

Under our topic of extended envelopes we should probably include most of the other variable stars since, except for the eclipsing stars, variations in light and spectrum are related to changes in the atmospheric parameters. We usually consider that any eruptions, major or minor, must pass through the outer, extended atmospheres which themselves are involved, even though the ongoing radiation may arise from interaction with a ring, disk, shell, envelope, circumstellar envelope or dissipation into the interstellar medium. Nearly all types of variable stars have members that are components of binary or multiple systems. In some cases, the possibility that the variability may be directly related to the duplicity is becoming accepted. We shall mention a few types of variables that come under the topic of extended atmospheres; the time spent on further discussion will depend on the interests of participants. Perhaps I should note here that while most variable stars have been discovered from their observed light variations, detailed spectroscopic analysis frequently discloses changes in their spectra. In most cases variable stars are rather faint. High-dispersion studies will undoubtedly prove most fruitful as the necessary telescopes and instrumentation become available.

Early-type stars, including Wolf-Rayet stars, shell stars, etc. have been discussed earlier in this review. Contact binaries have been discussed in previous sessions though I should like to mention Larsson-Leader's paper (1971) given at the Bamberg Colloquium last year in which he discusses the effects on a double-star system of gravitational fields, radiation flux and gas streams. I have barely touched on these effects which affect light curves as well as spectra, and which are now being studied very effectively, especially by Hill and Hutchings (1970, 1971). Fitch (1969) has considered gravitational and tidal effects on a pulsating star and related the phenomena to $\beta$ Cephei, $\delta$ Scuti and RR Lyrae stars.

The Cepheid variables certainly have extended atmospheres - and some 15 per cent of them are in binary systems (Lloyd Evans, 1968). Extensive theoretical investigations of shock-wave phenomena related to the RR Lyrae stars and the Cepheids have been made by Christy (1967) and the observational results have been summarized by Kraft (1967). Intensive investigations of southern Cepheids have been made by Bell and Rodgers $(1964,1967,1968)$. It has recently been suggested (Kraft, 1967) that at minimum radius the atmosphere corresponds to an F-type supergiant and that as the shock wave moves outward, the characteristics shift to those of later type.

The long-period variable stars undoubtedly have extensive atmospheres and, among 
others, Odgers and Kushwaha (1960) have proposed that a weak shock wave travelling through the atmosphere could produce the radial-velocities observed for $\mathrm{R}$ Hydrae over its light cycle; for this star there does not seem to be any mass loss, but the ejected material falls back on the star. Maehara (1971) has recently studied the velocities of the two components of the absorption lines in the spectrum of $\chi$ Cygni, observed particularly in the infrared. The long wavelength components seem to be formed in the outer, low-temperature region in front of the shock wave; the short wavelength components are formed in a higher-temperature region immediately behind the shock front which moves outward; the emission lines in the violet region of the spectrum are also formed behind the shock. Many of these lines were explained by Thackeray (1937) in terms of fluorescent, selective-excitation processes involving lines situated very close to strong MgII, FeII and SiI lines between $2000 \AA$ and $3000 \AA$; emission lines such as Fe I, $\lambda \lambda 4202$ and 4308 were accounted for in this way. Willson (1972) has taken these results and derived possible electron densities and temperatures for these stars. As Thackeray (1937) noted, the structure of the hydrogen emission lines shows that they arise from fairly low levels in the atmosphere, below the regions forming the $\mathrm{TiO}, \mathrm{Fe} \mathrm{I}$ and $\mathrm{Ca}$ II absorption lines. Until the advent of large telescopes it was almost impossible to observe long-period variable stars at minimum light with sufficient dispersion to identify the many lines. However Merrill (1953) measured Palomar plates of $\chi$ Cygni, taken by Bowen and by Wilson very near minimum, and found some 60 emission lines most of which could not be identified until Herbig (1956) showed that they are selected lines of the AlH molecule arising from levels near the rotational predissociation cutoff, as observed at low pressures in the laboratory. These atmospheres remain a very fruitful field for further study.

The RV Tau stars have been studied by Preston $(1962,1964)$ following observations by Abt (1953) that the hydrogen lines appear in emission and double lines also appear near maximum light. Preston showed that the violet absorption components are formed in hotter regions than are the red components. He thought that an emitting layer lies over the layer that produces the violet absorption lines and that the hydrogen emission layer is below that which produces the red absorption lines, but all are produced in an extended atmosphere. In the spectrum of $U$ Monocerotis emission lines of $\mathrm{Ti}$ I ( 26554$)$ appear at rising light as does $\mathrm{HeI}(\lambda 5876)$ and $\mathrm{H} \alpha$, though the latter appears in emission at all phases.

Eruptive variables have been studied extensively by Smak (1971) who reviewed the field and listed previous surveys. He discussed novae, U Geminorum-type stars and nova-like objects and considers that the principal feature of all such variables is a gaseous disc rotating about the primary component. As these stars have been discussed earlier, and as they may not have extended envelopes such as we are considering here, we shall not develop this subject further.

Flare stars should be mentioned since they seem to be related to solar flares from considerations of their lifetimes as well as their optical and radio emissions. Recent surveys have been given by Haro (1968) and by Gershberg (1969). Most of these stars are UV Ceti stars, which are Me dwarfs. Of 25 known stars in this group, 19 are 
known to be binaries, though 14 of these are visual binaries. The UV Ceti stars seem to have large convective zones and therefore considerable magnetohydrodynamic activity, which produces the solar flares, might be expected for these stars. As Gershberg remarks, Poveda showed that convection must be strong in low-luminosity stars up to class $\mathrm{K} 1$, and Haro found flare activity in precisely the same classes. At the most recent Bamberg Colloquium Ambartzumian and Mirzoyan (1971) gave a further summary of recent studies of flare stars. They are considered to be very young stars, the T Tauri and UV Ceti groups being the youngest. All flare stars seem to be latetype dwarfs and a great many are observed in associations and clusters - which led Ambartzumian and Mirzoyan to speculate, from observations of the Pleiades, that nearly all dwarf stars pass through the flare stage in the course of their evolution. The youngest stars are G- or K-types and evolve into M-type stars as shown by their relative frequency in young, and older, clusters. The presence of ionized calcium and hydrogen emission lines in their spectra can be taken as indicators of extreme chromospheres in these stars.

In summary, let us remind ourselves of conditions to be expected for observations of extended atmospheres. Immediately we talk about extended atmospheres, we imply that they are different from those of 'normal' stars. For about twenty years it was thought that spectra of normal stars might be explained and computed by model atmosphere techniques assuming local thermodynamic equilibrium and the Saha and Boltzmann equations relating temperature, pressure and ionization equilibria. The theoreticians now feel that non-LTE may be important for at least some lines even in normal stellar atmospheres. However for the Sun and similar stars, such effects are relatively small. Therefore we feel justified in assuming that gross deviations from the simple first approximations are indicators of extended atmospheres.

Observational criteria that have been used to detect extended atmospheres include: (i) geometrical effects: emission lines in stars of all types; (ii) velocity fields: broad, steep-sided absorption lines that can be recognized as having shapes different from the Stark-broadened wings of the hydrogen lines, the bell-shaped profiles of rotating stars, and the Voigt damping profiles of saturated lines; these are indicators of macroturbulence. Microturbulence can be detected from the gradient effect, first observed by Struve and Elvey (1934), where the weak lines of a multiplet are strengthened relative to the strong lines by curve-of-growth effects; (iii) dilution effects, which result from an accumulation of atoms in metastable levels when the pressure is low.

The evidence for extended atmospheres from observations of stellar spectra is overwhelming, though most of it has been accumulated over less than thirty years as a result of intensive investigations using the best equipment available. The evidence for differences between extended atmospheres of normal stars, and those of stars belonging to multiple systems is very small. In most of the categories that have been discussed here, there seem to be both single and multiple stars and there is very little evidence that there are major differences in the chromospheres resulting from the presence of another star. Even in the $\zeta$ Aurigae stars, where the effects can be analyzed 
best, the theory has not been developed sufficiently to decide about the effect of the hot B star on the atmosphere of its companion, which is less than $10 \mathrm{AU}$ away. There is evidence from the radial velocities that the chromosphere is not moving with a uniform rotation, such as might be expected for stars in binary systems, but the velocities do not suggest that material is moving systematically towards the secondary star. The temperature does seem to increase outwards for these stars, but there is a similar increase in the outer layers of the Sun. Thus data obtained from the chromospheres of components of double stars may give important information about the atmospheres of normal stars - and vice versa. Giant components of the $\zeta$ Aur stars seem to be the most suitable stars for such studies, as they are less affected by the secondary components and do not seem to have additional envelopes or shells.

It would appear that further investigations to compare the chromospheres of single stars with those in multiple systems would be a worthwhile and challenging task. Perhaps some of the participants at this Symposium have some of the answers.

\section{References}

Abt, H. A. : 1953, Astror. J. 58, 210.

Adams, W. S. and Russell, H. N.: 1928, Astrophys. J. 68, 9.

Ambartzumian, V. A. and Mirzoyan, L. V.: 1971, Veröffentl. Remeis-Sternw., Bamberg 9, 98.

Bell, R. A. and Rodgers, A. W.: 1964, Monthly Notices Roy. Astron. Soc. 128, 365.

Bell, R. A. and Rodgers, A. W.: 1967, Monthly Notices Roy. Astron. Soc. 138, 23.

Bell, R. A. and Rodgers, A. W.: 1968, Monthly Notices Roy. Astron. Soc. 139, 175.

Bidelman, W. P. and Pyper, D.: 1963, Publ. Astron. Soc. Pacific 75, 389.

Böhm-Vitense, E.: 1956, Publ. Astron. Soc. Pacific 68, 57.

Boyarchuk, A. A.: 1969, in L. Detre (ed.), Non-Periodic Phenomena in Variable Stars, D. Reidel, Dordrecht, p. 395.

Burbidge, G. R. and Burbidge, E. M.: 1953, Astrophys. J. 118, 252.

Chentsov, E. L. and Snezhko, L. I.: 1972, in M. Hack (ed ), Trieste Colloquium on Supergiant Stars, p. 51.

Christy, R. F.: 1967, in R. N. Thomas (ed.), 'Aerodynamic Phenomena in Stellar Atmospheres', IAU Symp. 28, Academic Press, London and New York, p. 105.

Conti, P.: 1972, Astrophys. J. Letters 174, L 79.

Cowley, A. P.: 1969, Publ. Astron. Soc. Pacific 81, 297.

Deutsch, A. J.: 1956, Astrophys. J. 123, 210.

Deutsch, A. J.: 1970, in D. C. Morton (ed.), 'Ultraviolet Spectra and Ground-based Observations', IAU Symp. 36, D. Reidel, Dordrecht, p. 317.

Doherty, L. R.: 1971, Phil. Trans. Roy. Soc. London A 270, 189.

Faraggiana, R. and Hack, M.: 1969, 'Les Transitions Interdites dans les Spectres des Astres', Mem. Soc. Roy. Sci. Liège, Sème Série 17, 317.

Feast, M. W.: 1970, in D. C. Morton (ed.), 'Ultraviolet Spectra and Ground-based Observations', IAU Symp. 36, D. Reidel, Dordrecht, p. 187.

Fitch, W. S.: 1969, in L. Detre (ed.), Non-Periodic Phenomena in Variable Stars, D. Reidel, Dordrecht, p. 287.

Gershberg, R. E.: 1969, in L. Detre (ed.), Non-Periodic Phenomena in Variable Stars, D. Reidel, Dordrecht, p. 111.

Ghobros, R. A.: 1962, Z. Astrophys. 56, 113.

Goedicke, V. A.: 1938, Publ. Univ. Michigan Obs. 8, 1.

Griffin, R. F.: 1963, Observatory 83, 255.

Groth, H. R.: 1957, Z. Astrophys. 43, 185.

Groth, H. R.: 1955, Z. Astrophys. 37, 261.

Groth, H. R.: 1961, Z. Astrophys. 51, 206, 231. 
Groth, H. R.: 1970, in H. G. Groth, and P. Wellmann (eds.), Spectrum Formation in Stars with Extended Atmospheres, NBS Spec. Publ. 332, p. 259.

Haro, G.: 1968, in Stars and Stellar Systems 5, 141.

Herbig, G. H.: 1956, Publ. Astron. Soc. Pacific 68, 204.

Herzberg, G.: 1948, Astrophys. J. 107, 94.

Hill, G. and Hutchings, J. B.: 1970, Astrophys. J. 162, 265.

Hutchings, J. B.: 1970, Monthly Notices Roy. Astron. Soc. 147, 161.

Hutchings, J. B.: 1970, in A. Slettebak (ed.), Stellar Rotation, D. Reidel, Dordrecht, p. 283.

Hutchings, J. B. and Hill, G.: 1971, Astrophys. J. 166, 373.

Hutchings, J. B. and Wright, K. O.: 1971, Monthly Notices Roy. Astron. Soc. 155, 203.

Kawabata, S.: 1957, Publ. Astron. Soc. Japan 9, 72.

Kitamura, M.: 1967, Publ. Astron. Soc. Japan 19, 194.

Kondo, Y., Giuli, R. T., Modisette, J. L., and Rydgren, A. E.: 1972, Astrophys. J. 176, 153.

Kraft, R. P.: 1960, in Stars and Stellar Systems 6, 370.

Kraft, R. P.: 1967, Astrophys. J. 150, 551.

Kraft, R. P.: 1967, in R. N. Thomas (ed.), 'Aerodynamic Phenomena in Stellar Atmospheres', IAU Symp. 28, Academic Press, London and New York, p. 207.

Larsson-Leander, G.: 1957, Stockholm Obs. Ann. 19, No. 8.

Larsson-Leander, G.: 1971, Veröffentl. Remeis-Sternw. Bamberg 9, 185.

Liller, W.: 1968, Astrophys. J. 151, 589.

Linsky, J. L.: 1968, Smithsonian Inst. Spec. Report, No. 274.

Linsky, J. L.: 1973, in S. D. Jordan and E. H. Avrett (eds.), Stellar Chromospheres, NASA SP-317, p. 48.

Lloyd Evans, T.: 1968, Monthly Notices Roy. Astron. Soc. 141, 109.

McKellar, A., Odgers, G. J., Aller, L. H., and McLaughlin, D. B.: 1952, Nature 169, 990.

McKellar, A., Aller, L. H., Odgers, G. J., and Richardson, E. H.: 1959, Publ. Dominion Astrophys. Obs. 11, 35.

Maehara, H.: 1971, Publ. Astron. Soc. Japan 23, 503.

Magnan, C.: 1965, Ann. Astrophys. 28, 512.

Menzel, D. H.: 1931, Publ. Lick Obs. 17, 1.

Merrill, P. W.: 1953, Astrophys. J. 118, 453.

Merrill, P. W.: 1960, in Stars and Stellar Systems 6, 509.

Merrill, P. W. and Sanford, R. F.: 1944, Astrophys. J. 100, 14.

Merrill, P. W. and Wilson, O. C.: 1956, Astrophys. J. 123, 392.

Odgers, G. J. and Kushwaha, R. S.: 1960, Publ. Dominion Astrophys. Obs. 11, 253.

Pannekoek, A. and Minnaert, M. J. G.: 1927, Proc. kungl. Akad. Weten. Amsterdam 30, 921.

Peery, B. F.: 1966, Astrophys. J. 144, 672.

Plavec, M.: 1968, Adv. Astron. Astrophys. 6, 201.

Praderie, F.: 1970, in H. G. Groth and P. Wellmann (eds.), Spectrum Formation in Stars with SteadyState Extended Atmospheres, NBS Spec. Publ. 332, p. 241.

Preston, G. W.: 1962, Astrophys. J. 136, 866.

Preston, G. W.: 1964, Astrophys. J. 140, 173.

Rosendahl, J. D.: 1970, Astrophys. J. 159, 107.

Sahade, J.: 1960, in Stars and Stellar Systems 6, 466.

Saito, M.: 1970, Publ. Astron. Soc. Japan 22, 455.

Smolinski, J.: 1972, in M. Hack (ed.), Trieste Colloquium on Supergiant Stars, p. 68.

Smak, J.: 1971, Veröffentl. Remeis-Sternw. Bamberg 9, 248.

Spitzer, L.: 1939, Astrophys. J. 90, 494.

Swings, J. P.: 1969, Astrophys. J. 155, 515.

Struve, O.: 1944, Astrophys. J. 99, 170.

Struve, O. and Elvey, C. T.: 1934, Astrophys. J. 79, 409.

Struve, O. and Roach, F.: 1939, Astrophys. J. 90, 727.

Thackeray, A. D.: 1937, Astrophys. J. 86, 499.

Thomas, R. N.: 1970, in H. G. Groth and P. Wellmann (eds.), Spectrum Formation in Stars with Extended Atmospheres, NBS Spec. Publ. 332, p. 259.

Thomas, R. N. and Athay, R. G.: 1961, Physics of the Solar Chromosphere, Interscience Publ. Co., New York. 
Thomas, R. N. and Gebbie, K. B.: 1971, Menzel Symposium, NBS Spec. Publ. 353, p. 84.

Walker, M. F.: 1972, Astrophys. J. 175, 89.

Willson, L. A.: 1972, Astron. Astrophys. 17, 355.

Wilson, O. C.: 1960, in Stars and Stellar Systems 6, 436.

Wilson, O. C.: 1966, Science 151, 1487.

Wilson, O. C. and Abt, H. A.: 1954, Astrophys. J. Suppl. Ser. 1, 1.

Wilson, O. C. and Aly, M. K.: 1956, Publ. Astron. Soc. Pacific 68, 149.

Wilson, O. C. and Bappu, M. K. V.: 1957, Astrophys. J. 125, 661.

Wright, K. O.: 1959, Publ. Dominion Astrophys. Obs. 11, 77.

Wright, K. O.: 1970, in A. Beer (ed.), Vistas in Astronomy 12, Pergamon Press, Oxford, p. 147.

Wright, K. O. and Odgers, G. J.: 1962, J. Roy. Astron. Soc. Can. 56, 149. 